\title{
Motion Calculation for Human Lower Extremities based on EMG-Signal-Processing and simple Biomechanical Model
}

\author{
Christian Fleischer, Konstantin Kondak, Christian Reinicke, and Günter Hommel ${ }^{1}$ \\ Technische Universität Berlin \\ \{fleischer, kondak, reinicke, hommel\}@cs.tu-berlin.de
}

Summary. In this paper we present a way to calculate the motion of the human lower extremities online based on surface EMG signals emitted by the activated muscles. The EMG signal evaluation is directly integrated into the control loop of the model to allow for more flexible and spontaneous movements than with predefined trajectories. The algorithm implements a simple biomechanical model composed of bones and muscles. An additional stability controller modifies the torques in the ankle, knee and hip joints to keep the model in a stable pose. The proposed method will be part of a control system for an exoskeleton robot where the movement of the model will be interpreted as the intended movement of the operator. The performance of the presented method was investigated on the stand-to-sit movement.

\section{Introduction}

For many decades surface electromyography has been studied by many researchers in the medical and biomechanical fi elds to get a deeper understanding of muscles and how and when they are activated.

In recent years more and more studies have explored the relationship between single muscles and the complex movements of the human body, but most of these studies were focused on analysing disabilites, anomalies and how to track progress in rehabilitation. Only a few publications focused on using electromyographical signals in real-time to control biomechanical robots e.g. [1-3].

The main reason is the diffi culty to map the EMG signal into the force a muscle is producing [4]. The approximated relationship itself is not too complex [5], but influenced by many different parameters. Some of these parameters only vary among different subjects, but some are even different from day to day. With the approach presented in [6] it is possible to calibrate the EMG-to-force relationship in a simple environment.

In this work we explain how calibrated muscle signals can be combined with motion controlling algorithms to achieve stable movements of a biomechanical model that reflects the intended movement of the operator. It is important to keep in mind, that this intention has to valid only for a short period of time in the future (fractions 
of a second). After that, the model can be synchronized with the reference system (synchronization is not done in this work to show the full effect of the model).

\subsection{Why EMG?}

Many different approaches for tracking the movement of a human being exist. Some use ultrasonic or visual sensors, some goniometers, accelerometers or other techniques. Every technique has its advantages and disadvantages, but all of the mentioned systems sense the current movement. With EMG signals it is possible to track the intended movement, which might differ from the current movement due to obstacles or lack of suffi cient muscle-power. And if the EMG sensors are placed carefully, the intention should even be readable ahead of time. The reason for this is that EMG signals are detectable slightly before the movement is performed, because muscles take some time to produce the force after having received the activation signal.

\section{Environment}

In this section the environment is described in which the biomechanical model and the motion controller is embedded. Refer to Fig. 1 for an initial overview of the whole system.

As stated before, the basic idea is to let the Human interact with the Mechanical System. To achieve this, EMG Signals are collected from some of the muscles of the subject and converted to forces in the block EMG-to-Force Converter. The resulting Forces are fed into the Biomechanical Model that represents the human subject. Through forward dynamics computation accelerations for all body parts are calculated from the muscle forces and the current state of the model. After doubleintegrating those accelerations, the resulting joint angles, velocities and accelerations form the new state of the model and are interpreted as the desired movement of the human. The Stability Controller analyses the model state and calculates supporting torques for the ankle, knee and hip joint to bring the model back into a stable pose if necessary. The supporting torques are fed back to the biomechanical model to take effect.

The Motion Controller takes the desired movement and computes the control signals for the Mechanical System. Because of the connection between the human body and the mechanical system, the motion of the actuators of the mechanical system affect the human body (Force Feedback). The Motion Controller and the Mechanical System are not yet fi nished and not part of this work.

To be able to calibrate the parameters of the EMG-to-Force Converter the calculated Kinematic Data from the Biomechanical Model are compared with the Reference Kinematic Data taken from the human body with additional sensors (see sec. 2.2). The computed parameters are then brought into the EMG-to-Force Converter again. Those additional sensors should be attached to the human with or without an exoskeleton to allow recalibration whenever necessary. The blocks Biomechanical Model and Stability Controller are the main focus of this work. 


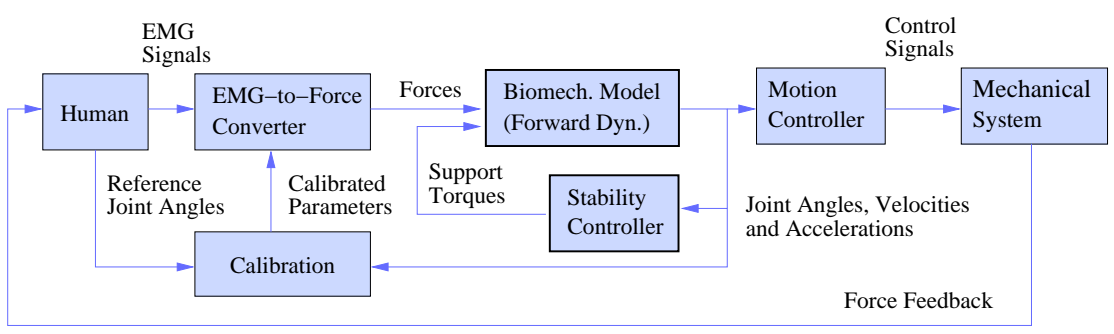

Fig. 1. Control scheme for a mechanical system attached to the human body: The EMG signals are captured from the subject, converted into forces and brought into the biomechanical model. Resulting joint angles and velocities from the model are passed to the motion controller to be executed by the mechanical system. The stabilty controller analyses the model state and computes supporting torques to keep a balanced pose. The calibration compares the joint reference angles with the angles computed by the model and modifies the EMG-to-force-parameters.

\subsection{EMG Setup}

The EMG signals are sampled with $1 \mathrm{KHz}$ from DelSys 2.3 Differential Signal Conditioning Electrodes [7] with an inbuilt gain of $1000 \frac{\mathrm{V}}{\mathrm{V}}$ and a bandpass fi lter from 20-450 Hz. The input data are rectifi ed and then smoothed by a lowpass fi lter with a cutoff frequency of $5 \mathrm{~Hz}$ [8].

\subsection{Reference System}

The reference system is needed to capture the actual movement of the human limbs for the calibration step. We have used a reference system based on the two axis accelerometers ADXL210 from AnalogDevices Inc. [9].

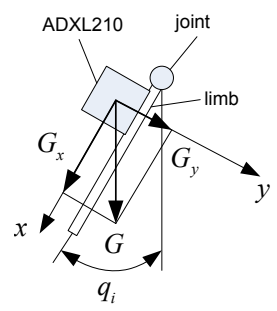

Fig. 2. Capturing the limb movement with two axes accelerometer ADXL210.

As shown in Fig. 2 the orientation of the limb in the sagittal plane can be calculated by projecting the earth gravity field into the $x$ - and $y$-axis of the sensor: $q_{i}=\arctan 2\left(G_{y i} / G_{x i}\right)$. One sensor was placed on each of the torso, thigh and shank and as close as possible to the rotation axis of the joints to reduce the inertial acceleration resulting from limb movement (the error is small enough below $45 \frac{\mathrm{deg}}{\mathrm{s}^{2}}$ ). Due to the nature of the sensors, there is only $0.5 \%$ temperature drift and a peak-to-peak noise below $2 \%$. 


\subsection{Biomechanical Model}

In literature a lot of information can be found about the anatomy of the human body: The properties of muscles, joints, tendons and tissues are available [10] and elaborated investigations have been done on biomechanical modelling of those [11]. Unfortunately, with increasing complexity of the model, more parameters have to be calibrated for every subject. Even though it is possible to create a complex model of the lower extremities [12], it is impossible to provide all those muscles in the model with properly recorded EMG values. This would result in an uncompensated defect of some muscles. So an equal layer of abstraction has to be applied everywhere.

For our experiments we have chosen a very simple model: It consists of the trunk, thigh, shank, foot and four muscles, as shown in Fig. 3 (both legs are modelled as one, since in our experimental setup they move as one). Each of those muscles in our model is representing a collection of muscles in the human body and does not necessarily have anatomical analogy. For the EMG signal acquisition we selected the muscles by their contribution to the most important movements of the leg: $\mathrm{m}$. sartorius $(M 0), \mathrm{m}$. glutaeus maximus $(M 1), \mathrm{m}$. quadriceps femoris $(M 2)$, and $\mathrm{m}$. semimembranosus (M3).

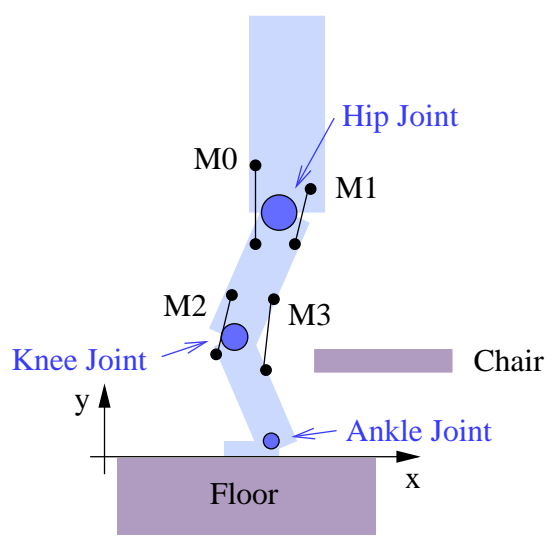

Fig. 3. The biomechanical model composed of trunk, thigh, shank, foot and four mucles: $M 0$, $M 1, M 2, M 3$.

We are aware that this is a rough approximation, but since the goal is to provide input values for a control unit of a mechanical construction and not pursueing clinical analysis, this seems to be an adequate simplifi cation. If required, more muscles can easily be integrated into the model.

Each of those muscles is only represented by a contractile element. The passive elastic and viscous elements are summed up in the joints spanned by the muscle (in contrast to the Hill model) together with the friction of the joints. This simplifi cation is similar to one presented in [13]. The overall frictions are assumed to be $5.0 \frac{\mathrm{Nms}}{\mathrm{rad}}$ (hip 
joint), 1.0 $\frac{\mathrm{Nms}}{\mathrm{rad}}$ (knee joint) and $0.5 \frac{\mathrm{Nms}}{\mathrm{rad}}$ (ankle joint). All values are inspired by [14] and were optimized by hand. The parameters of one muscle in our model are: the point of origin and insertion and the two parameters in the EMG-to-force function:

$$
F_{E M G}\left(\phi_{s}, \phi_{e}\right)=\phi_{s}\left(1-e^{-\phi_{e} x(t)}\right)
$$

where $x(t)$ is the postprocessed EMG signal and $\phi_{s}, \phi_{e}$ two muscle parameters. Since muscle origin and insertion are similar for the same average adult, offline optimization or calculation derived from the body dimensions can be used. Due to lack of space here, the model can be received from the authors on request. There are many other anatomical properties described in literature, but they are neglected for now.

Torso, thigh, shank and foot are modelled as cylindrical rigid bodies. The masses are calculated as fi xed fractions of the total body weight $\left(m_{\text {otal }}=88 \mathrm{~kg}\right)$ of the subject (fi gures can be found e.g. in [15]). The dynamic equations were derived using Kane's formalism [16], and have the following form:

$$
\mathbf{M}(\mathbf{q}) \dot{\mathbf{u}}=\mathbf{f}(\mathbf{q}, \mathbf{u})+\mathbf{T}(\mathbf{q}, \mathbf{u})
$$

where

- $\mathbf{q}=\left(q_{\text {ankle }}, q_{\text {knee }}, q_{h i p}\right)^{T}$ is the vector of generalized coordinates (joint angles)

- $\mathbf{u}=\left(u_{\text {ankle }}, u_{\text {knee }}, u_{\text {hip }}\right)^{T}$ is the vector of corresponding generalized velocities (with $\dot{\mathbf{q}}=\mathbf{u}$, the dot denotes the time derivative in a Newtonian reference frame)

- $\mathbf{M}(\mathbf{q})$ (matrix function) takes into account the mass distribution

- $\mathbf{f}(\mathbf{q}, \mathbf{u})$ (vector function) describes the influence of both inertial forces and gravity

- $\mathbf{T}$ is a vector of the generalized forces applied to the system. For the model considered, these are:

- the forces produced by the muscles $\left(F_{E M G}\right)$ multiplied with the nonlinear function $\mathbf{g}(\mathbf{q})$ (current system confi guration and geometry of the muscles)

- friction torques in joints (depending on $\mathbf{u}$ )

- supporting-torques vector $\mathbf{t}_{\mathbf{s}}=\left(t_{\text {ankle }}, t_{\text {knee }}, t_{\text {hip }}\right)^{T}$ that keeps the model in a stable pose (additional torques in the joints)

- temporary contact force $\mathbf{f}_{\mathbf{c}}$

For the experiments perfomed (see sec. 4) an additional external force $\mathbf{f}_{\mathbf{c}}$ is needed that must be applied to the hip to simulate the contact force with the chair when sitting down. A small P-controller simulates the effect of the chair contact force. If the $y$-coordinate of the hip $y_{\text {hip }}$ moves below a certain value $y_{\text {chair }}$ (height of the chair) the contact force $\mathbf{f}_{\mathbf{c}}$ is applied to the hip to move the hip back on top of the chair and to the first point of contact with the chair in $x\left(x_{c c}\right)$ :

$$
\mathbf{f}_{\mathbf{c}}= \begin{cases}\text { with }\left(\left(x_{c c}-x_{\text {hip }}\right) * \alpha,\left(y_{\text {chair }}-y_{\text {hip }}\right) * \beta, 0\right)^{T} & \text { if } y_{\text {hip }} \leq y_{\text {chair }} \\ (0,0,0)^{T} & \text { otherwise }\end{cases}
$$

where $y_{\text {chair }}=0.45 \mathrm{~m}, \alpha=1.5 \times 10^{5} \frac{\mathrm{N}}{\mathrm{m}}, \beta=1.0 \times 10^{5} \frac{\mathrm{N}}{\mathrm{m}}$ (experimentally determined). This controller might be substituted by an external sensor (either to get a boolean value for "contact" / "no contact" or the force value $f$ ). 
An external floor contact force is not needed since the foot position is fi xed and the reaction forces can be calculated implicitly through the model. The dynamic eqs. (2) were generated with the symbolic manipulation tool AUTOLEV [17]. The script for the system description and eqs. generation can be received on request.

\section{Stability Controller}

The stability controller is activated whenever the recorded EMG signals are not suffi cient to keep the model in a stable pose. $f_{M 1}$ (force of muscle group $M 1$ ) is monitored and whenever $f_{M 1}<500 \mathrm{~N}$ the stability controller is activated. In our experiments this is the case when standing upright. The controller is based on an approach presented in [18]. It takes the current state of the model $(\mathbf{q}, \mathbf{u}, \dot{\mathbf{u}})$ as and input and calculates the supporting-torques vector $\mathbf{t}_{\mathbf{s}}$ that is applied to the joints. The stabilty condition is

$$
x_{c m}=x_{c} \text { with } x_{c}=\frac{x_{t o e}+x_{\text {heel }}}{2}
$$

Where $x_{c m}$ is the $x$-coordinate of the center of mass of the whole body, $x_{\text {toe }}$ the $x$ coordinate of the tip and $x_{\text {heel }}$ of the heel of the foot. From the dynamic equations of the model $x_{c m}$ can be rewritten as

$$
x_{c m}=g(\mathbf{q})
$$

where $g$ is a non-linear function in $\mathbf{q}$. Dual differentiation of eq. 4 leads to

$$
a_{c m}=\beta_{0}+\beta_{1} \dot{u}_{\text {ankle }}+\beta_{2} \dot{u}_{k n e e}+\beta_{3} \dot{u}_{h i p}
$$

where $\beta_{0}, \cdots, \beta_{3}$ are non-linear functions in $\mathbf{q}$ and $\mathbf{u}$. If $\dot{\mathbf{u}}$ is chosen in a way that eq. 5 is satisfi ed the center of mass $x_{c m}$ is moving towards $x_{c}$. This can be calculated by interpreting eq. 5 as a plane in the acceleration space where $\beta_{0}, \cdots, \beta_{3}$ are considered constants for a given moment in time (for a detailed explanation, see [18]) and $\dot{\mathbf{u}}$ as variables with arbitrary values. Projecting $\dot{\mathbf{u}}_{d e s}$ (necessary acceleration to reach a desired pose) onto the plane defi ned by $\beta_{1}, \beta_{2}, \beta_{3}$ and $\beta_{0}-a_{c m}$ we get the support accelerations $\dot{\mathbf{u}}_{\mathbf{s}}$ and through inverse dynamic calculation the supporting torques $\mathbf{t}_{\mathbf{s}}$. $\dot{\mathbf{u}}_{\mathbf{d}}$ is

$$
\dot{\mathbf{u}}_{\mathbf{d}}=k *\left(q_{\text {ankle,des }}-q_{\text {ankle }}, q_{k n e e, d e s}-q_{k n e e}, q_{\text {hip }, \text { des }}-q_{\text {hip }}\right)
$$

where $k=100 \frac{\mathrm{Nm}}{\mathrm{s}^{2}}$. For the calculation of the desired coordinates $q_{\text {ankle,des }}, q_{\text {knee,des }}$ (standing position) the hip is at $y_{h i p}=0.955 \mathrm{~m}$ above the ankle and $q_{h i p}$ is calculated by solving eq. 4 with $x_{c m}=x_{c}$.

\section{Experiments}

The experiments were started in an upright standing position of the subject with both legs parallel. The subject was sitting down on a chair in a natural way (see Fig. 4). 

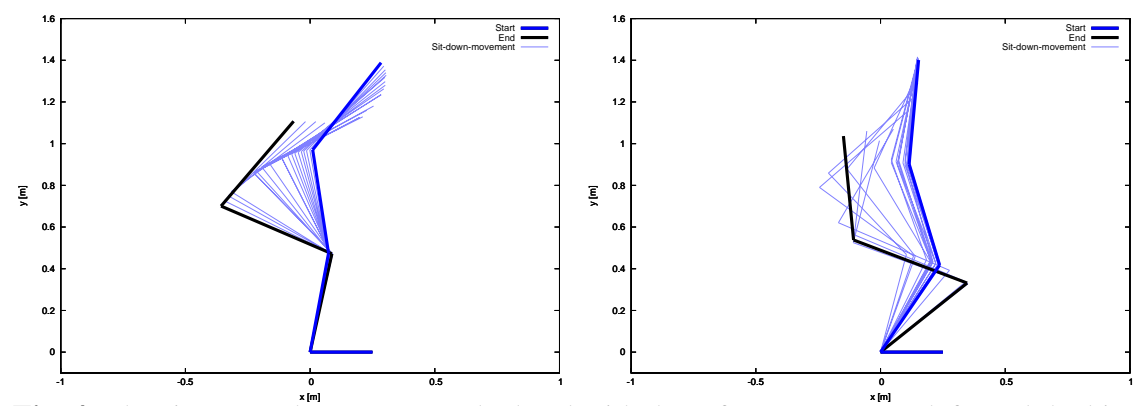

Fig. 4. The sit-to-stand movement calculated with the reference sensors (left) and the biomechanical model (right). In between a good correlation of the movement can be seen. Distortions at the start are due to different stability conditions and missing masses (head, arms) in the model. Because no muscle for bending the back or the ankle is recorded, the hip is not pushed back (and the back is straight). This also affects the resulting knee and ankle angles.

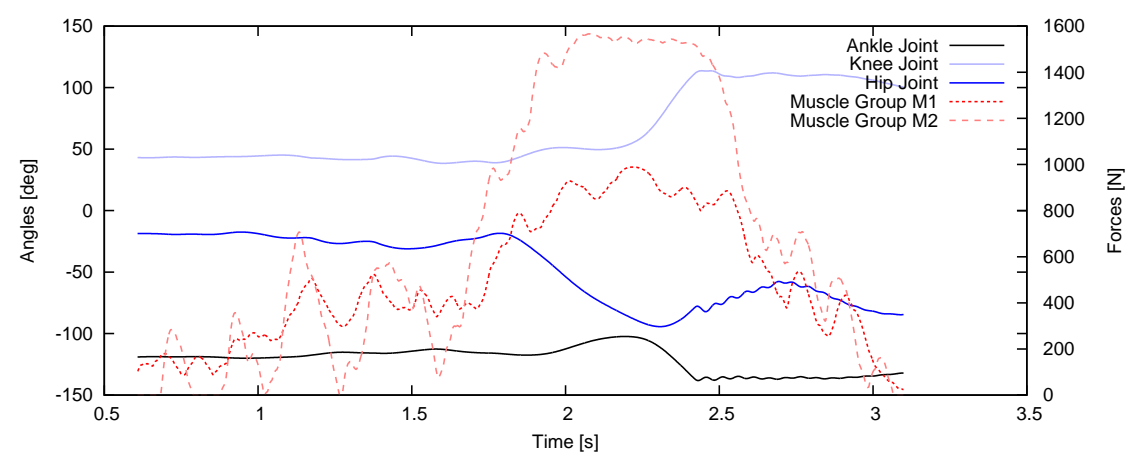

Fig. 5. Angles in the ankle, knee and hip joints during the movement are shown with the muscle forces here. Notice how the hip angle is changing when group $M 1$ gets stronger. $M 0$ is especially strong when the knee is bent. The ankle joint is only modified passively through the other joints.

During the experiment, the main force contributions from the modelled muscles come from the groups represented by $M 1$ and $M 2$. The only external forces affecting the motion was gravity and the chair contact force $f_{c}$ (only temporary).

Fig. 5 shows the angles in the ankle, knee and hip joints during the movement together with the muscle forces to show the interaction of the muscle forces with the modelled body parts.

\section{Discussion and conclusion}

As can be seen in this experiment, it is possible to calculate the intended movement of the subject with EMG signals. Although the presented movement here is quite simple compared to real-life situations, it should not be forgotton that for analysing the intentions of a subject the biomechanical model has to be valid only for a fraction 
of a second after synchronisation with the reference system. In the middle of the movement the model imitates the reference quite good. To use the algorithm as a versatile intention reader for more complex movements, a lot of work needs still to be done. More muscles have to be integrated (at the torso and shank) and the stabilty controller has to be more flexible. Also the issue of force feedback from the exoskeleton will be a major topic for further research. But the results so far look promising for future work.

\section{References}

1. S. Lee and G. N. Sridis, "The control of a prosthetic arm by EMG pattern recognition," IEEE Transactions on Automatic Control, vol. AC-29, no. 4, pp. 290-302, 1984.

2. O. Fukuda, T. Tsuji, H. Shigeyoshi, and M. Kaneko, "An EMG controlled human supporting robot using neural network," in Proceedings of the IEEE/RSJ Int. Conf. on Intelligent Robots and Systems, 1999, pp. 1586-1591.

3. S. Morita, T. Kondo, and K. Ito, "Estimation of forearm movement from EMG signal and application to prosthetic hand control," in Proceedings of the IEEE Int. Conf. on Robotics \& Automation, 2001, pp. 3692-3697.

4. J. V. Basmajian and C. J. De Luca, Muscles Alive: Their Functions Revealed by Electromyography. Williams \& Wilkins, 1985.

5. A. L. Hof, "EMG to force processing," Ph.D. dissertation, University of Groningen, 1980.

6. C. Fleischer, K. Kondak, C. Reinicke, and G. Hommel, "Online calibration of the emg to force relationship," in Proceedings of the IEEE/RSJ Int. Conf. on Intelligent Robots and Systems, 2004, p. about to appear.

7. DelSys, Inc., http://www.delsys.com.

8. M. Thompson, M. Voigt, and M. de Zee, "Evaluation of lower extremity musculo-skeletal model using sit-to-stand movement," in 19th congress of the International Society of Biomechanics, 2003.

9. Analog Devices, Inc., http://www.analog-devices.com.

10. N. Palastanga, D. Field, and R. Soames, Anatomy and Human Movement. Structure and Function. Butterworth Heinemann, 2002.

11. M. Epstein and W. Herzog, Theoretical Models of Skeletal Muscle: Biological and Mathematical Considerations. John Wiley \& Sons, Inc., 1998.

12. S. Delp, J. Loan, M. Hoy, F. Zajac, E. Topp, and J. Rosen, "An interactive graphics-based model of the lower extremity to study orthopaedic surgical procedures," IEEE Transactions on Biomedical Engineering, vol. 37, no. 8, pp. 757-767, Aug. 1990.

13. R. Riener, "Neurophysiological and biomechanical modeling for the development of closed-loop neural prostheses," Ph.D. dissertation, Techn. Universität München, 1997.

14. M. Günther, "Computersimulation zur Synthetisierung des muskulär erzeugten menschlichen Gehens unter Verwendung eines biomechanischen Mehrkörpermodells," Ph.D. dissertation, University of Tübingen, 1997.

15. D. A. Winter, Biomechanics and Motor Control of Human Movement. John Wiley \& Sons, Inc., 1990.

16. T. Kane and D. Levinson, Dynamics OnLine: Theory and Implementation with Autolev. Kane Dynamics, Inc., 2000.

17. Kane Dynamics,Inc., http://www.autolev.com.

18. K. Kondak and G. Hommel, "Control and online computation of stable movement for biped robots," in Proceedings of the IEEE/RSJ Int. Conf. on Intelligent Robots and Systems, 2003, pp. 874-879. 